is throughout concerned only with deviations of up to a few per cent at worst, because three variables are available for fitting, and the calculation is insensitive to the last binding step).

For the all-or-none, allosteric, model of Monod, Wyman and Changeux, using for the three adjustable parameters the equilibrium constant relating the high and low affinity states of the protein, the ratio of their ligand affinities and one of the two rate constants demanded by the model, the fit is again poor. It may be noted that the release of diphosphoglycerate after oxygenation of the third haem was earlier deduced by Tyuma et al. (Biochem. Biophys. Res. Commun., 43, 423; 1971), simply from a comparison of the parameters defining the oxygen dissociation profiles in terms of the Adair equation, in the presence and absence of the cofactor. They were able to show moreover that the last oxygenation step could be quantitatively identified with the reaction of an isolated $\beta$-chain.

These results then are without doubt strong arguments in favour of one type of model. At the same time, Hopfield et al. (J. Mol. Biol., 61, 425; 1971) have been at pains to show that assorted rate data garnered from the literature are at no point inconsistent with an allosteric formulation. The oxygen saturation rate curves are adequately fitted in such terms, though not as well as by a set of Adair constants. Hopfield et al. regard such curve fitting as suspect, however, and insufficient evidence in favour of a model. Their own preference is based essentially on the ability of the allosteric formalism to absorb published data on the changes in concentration of rapidly reacting haemoglobin with the extent of ligand dissociation caused by flash photolysis.

At a more utilitarian level, and as a postscript to my account recently of the use of cyanate and related compounds to inhibit the sickling reaction in sickle cell anaemia, there is a new study by Gillette, Manning and Cerami (Proc. US Nat. Acad. Sci., 68, 2791; 1971). These workers, who were responsible for the original (and successful) attempt to mitigate sickling by reacting the chain termini of the abnormal haemoglobin with cyanate, have now examined the effect of such modification on the survival of the red cells in the patient. Cells, labelled with isotopic chromium, were treated with cyanate and then returned to their owner. The half-population survival time increased from an average of 10 to 21 days, which compares much more favourably with the 20-35 days survival span of normal cells. The latter were essentially unaffected by cyanate ion. This result then may turn out to be one of the occasional triumphs of the biochemist over a baleful phenomenon of nature.

\title{
Ribonuclease and Reverse Transcriptase
}

FEw molecular biologists today would quarrel with the idea that infection of cells by RNA tumour viruses depends on the synthesis of a double stranded DNA molecule, the provirus, specified by the single stranded RNA genome, and that this synthesis is catalysed by reverse transcriptase. Baltimore and his colleagues have shown (Nature New Biology, 233, 131; 1971) that reverse transcriptase is similar to DNA polymerase I of Escherichia coli and requires a primer with a $3^{\prime}$ hydroxyl group on which to initiate DNA synthesis, and countless groups have by now shown that an RNA/DNA hybrid molecule and some single stranded DNA molecules are intermediary to the appearance of double stranded DNA. Mölling and five colleagues working in Tübingen now report in next Wednesday's Nature New Biology the discovery of a ribonuclease activity, RNAase $\mathrm{H}$, which seems to be responsible for the displacement of single stranded DNA from RNA/DNA hybrids.

Having recently isolated, from calf thymus cells, and RNAase which specifically digests the RNA strand of RNA/ DNA hybrids, Mölling and his associates searched for a similar sort of activity in avian myeloblastosis virus particles
(AMV) and sure enough they found it. Preparations of AMV particles disrupted with detergent will digest the RNA of RNA/DNA hybrids made either by transcribing calf thymus DNA with $E$. coli RNA polymerase or by annealing phage fd DNA with complementary RNA.

Mölling et al. then attempted to fractionate this RNAase $\mathrm{H}$ activity from the AMV virions but none of the several separation methods they used resulted in the separation of reverse transcriptase activity from RNAase $\mathrm{H}$ activity. Indeed the degradation of the single stranded RNA in the AMV particles by endogenous RNAase $\mathrm{H}$ was found to depend, at least in part, on the reverse transcription of this RNA. This discovery suggests that RNAase $\mathrm{H}$ activity is responsible for generating single stranded DNA during the synthesis of a provirus and it may well be that the polymerase and the ribonuclease activities reside in the same enzyme molecule. There is, of course, a precedent for this situation. DNA polymerase I of $E$. coli has both DNA polymerase and DNAase activities; the already considerable parallels between reverse transcriptase and DNA polymerase I may, therefore, extend still further.

\section{Surface Characteristics of B Cells}

Cellular immunologists are now devoting much effort to describing the heterogeneity of lymphocytes which, to the despair of the old-fashioned morphologist, look exactly alike. The efforts are directed partly to arrive at satisfactory counts of say B or T cells in various species, particularly man and, partly, to determine the functional attributes of lymphocytes. In next Wednesday's Nature New Biology two groups of immunologists, Fröland, Natvig and Berdal, and Dwyer, Mason, Warner and Mackay, report work which reflects these aims.

Fröland et al. have approached the problem of identifying B cells in man by the use of fluorescent anti-immunoglobulin antisera. Most theorists suppose that the capacity of B cells to be stimulated to antibody production depends initially on their possession of antibody-like receptors on the cell surface. Using a battery of more or less specific antisera conjugated with a fluorochrome marker, Fröland et al. found that up to 10 per cent of the peripheral blood lymphocytes from six normal patients showed visible fluorescence indicative of surface bound immunoglobulin. The monospecific antisera which were class or sub-class specific reacted with smaller percentages of cells. The marginal indication was that different cells carried different immunoglobulins on their surfaces. In contrast the blood lymphocytes from six patients with Bruton type agammaglobulinaemia, a complaint associated with failure to produce serum antibody, showed no staining with any antisera.

Wilson and Nossal using another technique (Lancet, ii, 788; 1971) have recently arrived at a figure of 34 per cent for the frequency of human lymphocytes with a high density of surface-bound immunoglobulin. The discrepancy between these results and those of Fröland et al. is probably indicative of a difference in sensitivity of the two techniques. But it is clear that with either method the surface-bound immunoglobulins are probably a sound characteristic of human B cells.

Dwyer et al. incubated spleen cells from nude mice, which are widely believed to be congenitally athymic, with a variety of different radioactively labelled antigens. They find that the proportions of labelled cells $(3-18 \times$ $10^{-4}$ ) were not significantly different between nude and normal mouse spleen cells, suggesting that only $\mathrm{B}$ cells are antigen binding cells (ABC as they say). In a sense this work dovetails nicely with the previous studies on immunoglobulins on the surfaces of B cells. 